\title{
The mechanism of biomedical therapy in the treatment of viral hepatitis.
}

\author{
Li-Jun Yu*, Zi-Ping Chen \\ Department of Liver Diseases, Qianfoshan Hospital Affiliated to Shandong University, Shandong University, Jinan, \\ 250012, PR China
}

\begin{abstract}
Background: Although there is a significant improvement in the living standards of people globally, a variety of disease outbreaks occurred over the time. Hepatitis B disease is contagious with a complex mode of transmission.

Methods: In this paper, the effects of biomedical therapy on the treatment of hepatitis B were studied.

Results: The mechanism of action was found, so as to provide a theoretical basis for the future application of biomedical therapy in the treatment of viral hepatitis.

Conclusions: The results show that the method is effective.
\end{abstract}

Keywords: Biomedical therapy, Viral hepatitis, Rehabilitation therapy, Mechanism.

Accepted on May 26, 2017

\section{Introduction}

Hepatitis B is a contagious and typical epidemic disease. It is caused by hepatitis b virus (HBV) and the way of transmission is very complex [1]. This disease also has the characteristics of high incidence and the spread of the disease in China is very extensive. On a global scale, China is a highly endemic area of hepatitis B [2]. The positive rate of hepatitis B patients in China is as high as $15 \%$, and the disease carriers are very common [3]. The clinical manifestations of hepatitis B are very complex, which may cause acute hepatitis, chronic hepatitis, liver cirrhosis and liver cancer, and in some cases it may lead to rapid death of the patients. Therefore, hepatitis B is very dangerous to human health.

In the field of medicine, the more common view is that hepatitis B is mainly due to the damage of liver cells and the immune system. But the HBV is not the direct cause for liver cell injury. Cytotoxic T lymphocytes (CTL) are mainly responsible for the damage of liver cells during hepatitis $\mathrm{B}$. The CTLs produce antiviral cytokines to kill the virus and in this process they damage the infected hepatocytes [4].

\section{State of the Art}

Hepatitis B disease has plagued millions of people for a long time by causing broad-spectrum of liver diseases such as hepatitis, cirrhosis and liver cancer. Hepatitis B is a kind of systemic infection, affecting the human body organs [5]. The hepatitis B symptoms include general malaise, bloating, nausea, dry stool and other symptoms. Hepatitis B is highly contagious and can be divided into many kinds, such as jaundice type and non-jaundice type, etc.
There are 120 million HBV carriers in China and they're all suffering with chronic HBV infection. The spread of hepatitis $\mathrm{B}$ in China is shown in Figure 1.

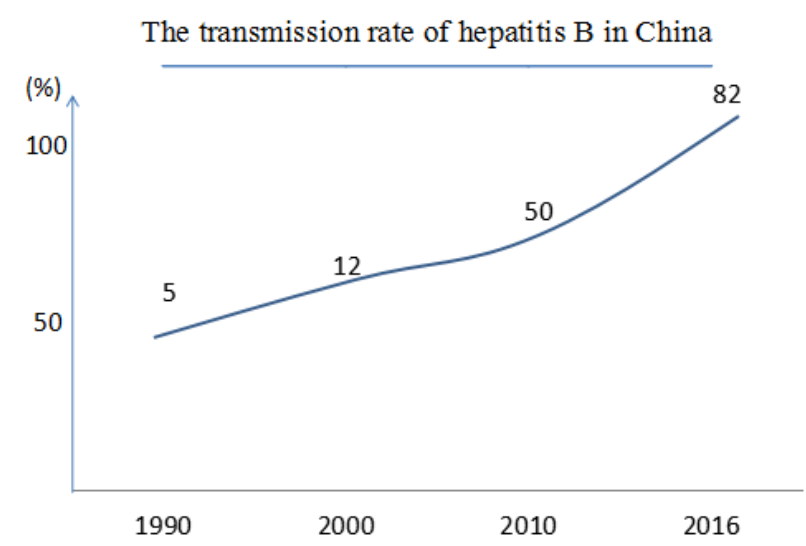

Figure 1. The transmission rate of hepatitis $B$ in China.

From the Figure 1, it is evident that almost all the people were infected with HBV either during the fetal period or early childhood. Even after more than ten years of infection, HBV is still persistent in their bodies, but does not cause hepatitis. Yet, these people can still continue to discharge HBV, which is an important source of hepatitis B infection. Hepatitis B replicates in the liver, but does not directly damage the liver cells; this has been confirmed in the case of $\mathrm{HBV}$ patients with hyperthyroidism and animal studies [4,6-8]. The formation of persistent HBV infection chronicity is due to persistent immune tolerance state induced by the virus; in particular, it is associated with the low reactivity of cytotoxic $\mathrm{T}$ cells. 


\section{Methodology}

\section{Biological therapy}

In this study, 30 patients with hepatitis B hepatitis were selected as the research objects. There were 20 cases of chronic hepatitis $\mathrm{B}$, including 5 cases of male and 15 cases of female. The average age was 30 years old, the shortest history of the onset was six months and the longest was 11 years. The average history was 6.5 years. In this study, 30 patients with hepatitis B were divided into group A and group B. Group A was treated with biological therapy, group B was treated with traditional medicine. The experiments were conducted twice in a day for both the groups and 1 drug was taken each time.

\section{Regulating effect of biological treatment on hepatitis $\boldsymbol{B}$}

In this study, double antibody sandwich method is used to detect serum tumor necrosis factor (TNF) which is a kind of cytokine involved in the body's immune system and has strong activity. TNF interacts with interferon and interleukin, so as to form a dynamic immune regulatory system. The excessive secretion of TNF can damage the mediating factor, and the TNF in the serum of normal people is obviously lower than that of the patients with hepatitis B. The reason for this phenomenon may be the effect of $\mathrm{HBV}$ on macrophages, which stimulates endotoxemia and immune complexes. In patients with hepatitis $\mathrm{B}$, the virus destroys $\mathrm{T}$ lymphocytes, which makes the function of lymphocytes changed. As a result of which, the cytotoxic T cells in hepatitis B patients are higher than those in normal subjects. The proportion of TNF, interleukin and interferon in healthy volunteers is shown in Figure 2.

\section{The proportion of TNF, interleukin and interferon in healthy volunteers}

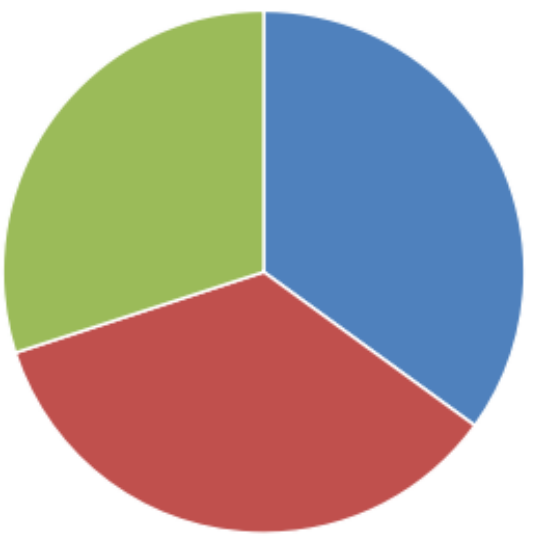

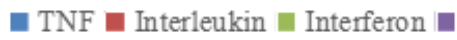

Figure 2. The proportion of TNF, interleukin and interferon in healthy volunteers.

Biological therapy has obvious therapeutic effect on patients with hepatitis B. It not only provides supplementation and protect the liver cells, but also can improve the liver function, and protect the immune system from invasion. Biological treatment also can reduce the abnormal rate of $\mathrm{T}$ lymphocyte subsets in patients. It can effectively remove the virus from the patient's body, and ultimately restore the effect of liver function. It can also enhance the body's immune function.

\section{Result Analysis and Discussion}

In this study, the liver function was observed through the ALT and $\mathrm{A} / \mathrm{G}$; the two had return to normal, which was called better. The detection indicators of hepatitis $\mathrm{B}$ included the HBsAg, anti-HBe, anti-HBs conversion. The improvement rate of the two groups of patients with clinical symptoms is shown in Table 1.

Table 1. Changes of liver function in two groups.

\begin{tabular}{lllll}
\hline & \multicolumn{3}{c}{ ALT } & A/G \\
\cline { 2 - 5 } & $\begin{array}{l}\text { Good } \\
\text { revolution }\end{array}$ & $\begin{array}{l}\text { Good } \\
\text { revolution }(\%)\end{array}$ & $\begin{array}{l}\text { Good } \\
\text { revolution }\end{array}$ & $\begin{array}{l}\text { Good } \\
\text { revolution (\%) }\end{array}$ \\
\hline Drug group & $7 / 30$ & $23.3 \%$ & $3 / 22$ & $13 \%$ \\
\hline $\begin{array}{l}\text { Holographic } \\
\text { group }\end{array}$ & $15 / 30$ & $50 \%$ & $8 / 20$ & $40 \%$ \\
\hline $\mathrm{P}$ & & 0.95 & & $\leq 0.65$ \\
\hline
\end{tabular}

Every part of the human body contains all the information of the whole body, and the traditional Chinese medicine (TCM) considers holographic embryo is equivalent to the human body [9]. A certain point on the holographic embryo is equivalent to an organ of the human body; there is a great similarity between the liver cells and the liver cells on the holographic embryo. For a point on the holographic embryo, the point will produce some damage. In response to that the body will produce antibodies against the damaged cells. The activated immune material not only repairs the damage site, but also repair the damage caused by the virus, and ultimately kill the virus. This is the mechanism of biological treatment of hepatitis B.

\section{Conclusions}

Hepatitis B has been a difficult problem for people at present. Hepatitis B is a systemic infection caused by HBV which damage the human organs. The clinical manifestations of hepatitis B are very complex, which may cause acute hepatitis, chronic hepatitis, liver cirrhosis and liver cancer, and in some cases it may lead to rapid death of the patients. Therefore, hepatitis B is considered as a dreadful infectious disease which is very dangerous to human health. Based on this, this paper studied the effect of biomedical therapy for the treatment of hepatitis B, and found out the mechanism, so as to provide some theoretical basis for the application of biomedical therapy in the treatment of viral hepatitis.

\section{References}

1. Pan CQ, Zhang JX. Natural history and clinical consequences of hepatitis b virus infection. Int $\mathrm{J}$ Med Sci 2005; 2 : 36-40. 
2. Feng-Min L, Hui Z. Management of hepatitis B in China. Chinese Med J 2009; 122: 3-4.

3. Yu J, Han J, Zhang J. The long noncoding RNAs PVT1 and uc002mbe. 2 in sera provide a new supplementary method for hepatocellular carcinoma diagnosis. Medicine 2016.

4. Oh IS, Park SH. Immune-mediated Liver Injury in Hepatitis B Virus Infection. Immune Netw 2015; 15: 191-198.

5. Wang ML, Tang H. Nucleos (t) ide analogues causes HBV $\mathrm{S}$ gene mutations and carcinogenesis. Hepatobiliary Pancreatic Dis Int 2016; 15: 579-586.

6. Chisari FV, Ferrari C. Hepatitis B virus immunopathogenesis. Annu Rev Immunol 1995; 13: 29-60.

7. Ferrari C, Missale G, Boni C, Urbani S. Immunopathogenesis of hepatitis B. J Hepatol 2003; 39: S36-S42.
8. Ganem D, Prince AM. Hepatitis B virus infection--natural history and clinical consequences. N Engl J Med 2004; 350: 1118-1129.

9. Yong Z. The universal holographic law and the model of the planet style of the human body. 4th World Conf Acad Exch Med Qigong, 1998.

\section{*Correspondence to}

Li-Jun Yu

Department of Liver Diseases

Qianfoshan Hospital Affiliated to Shandong University

Shandong University

PR China 